\title{
Semigroup Theory for Control on Sun-reflexive Banach Spaces
}

\author{
H. J. A. M. Heijmans \\ Centre for Mathematics and Computer Science, P.O. Box 4079, 1009 AB \\ Amsterdam, The Netherlands
}

[Received 23 September 1986]

We use the theory of dual $\mathrm{C}_{0}$-semigroups, as developed by Phillips, to define and study a new class of control systems on nonreflexive Banach spaces. Our main results concern the (approximate) controllability and observability of such systems. We illustrate our abstract results with an application to a delay system.

\section{Introduction}

AN important and large class of control systems can be described by

$$
\frac{\mathrm{d} x}{\mathrm{~d} t}(t)=A x(t)+B u(t), \quad x(0)=\varphi .
$$

Here $\varphi$ is an arbitrary point in the Banach space $X$, the operator $A$ is the infinitesimal generator of a strongly continuous semigroup (or $\mathrm{C}_{0}$-semigroup) $T(t)$ on $X$, and $B$ is a bounded linear operator from another Banach space $U$, the control space, into $X$. In (1.1), $\mathrm{d} x / \mathrm{d} t=A x$ describes the uncontrolled action, and the inhomogeneous term $B u(t)$ represents the control action. Many authors have studied systems of a more general form than (1.1). See for instance $[4,8,12,13]$. In all cases, their motivation was that (1.1) is too special for a number of applications. It does not cover systems described by partial differential equations, the control action of which is applied on the boundary: the so-called boundary control systems. Neither does it describe control systems with delay on the space of continuous functions. In this paper, we present a rather natural generalization of (1.1), which covers delay equations, and which looks promising with respect to boundary control systems. The keyword for our approach is duality, the main tool being the theory of dual $\mathrm{C}_{0}$-semigroups as it was developed by $\mathrm{R}$. S. Phillips, and described in [9: Ch. XIV].

A short non-rigorous description follows. It is well known that we can replace (1.1) by

$$
x(t)=T(t) \phi+\int_{0}^{t} T(t-s) B u(s) \mathrm{d} s .
$$

(1.2) defines so-called mild (i.e. continuous) solutions, which are not necessarily continuously differentiable. Below, we indicate that the integral still has a meaning for a larger class of operators $B$. We start with a brief exposition on the theory of dual $\mathrm{C}_{0}$-semigroups; for a more thorough treatment we refer to 
the following section. If $X$ is a nonreflexive Banach space, then $T^{*}(t)=T(t)^{*}$ defines a semigroup on $X^{*}$ which is not necessarily strongly continuous again: it is weak $*$ continuous, however. Let $X^{\odot}$ be the closed subspace of $X^{*}$ on which $T^{*}(t)$ is strongly continuous; then $X^{\odot}$ is invariant under $T^{*}(t)$, and by $T^{\odot}(t)$ we denote the restriction of $T^{*}(t)$ to $X^{\odot}$. Obviously, $T^{\odot}(t)$ is a $\mathrm{C}_{0}$-semigroup on $X^{\odot}$, and we can repeat our 'game' of taking duals and restrictions. Then $X$ lies continuously embedded in $X^{\odot *}$, and in some cases this embedding coincides with $X^{\odot \odot}=\left(X^{\odot}\right)^{\odot}$. If this is the case, we say that $X$ is $\odot$-reflexive (sun-reflexive) with respect to $A$.

Now assume that $X$ is $\odot$-reflexive with respect to $A$. For simplicity we identify $X$ with its embedding in $X^{\odot *}$ :

$$
X=X^{\odot \odot}
$$

In Section 2 , it is indicated that this can be done properly by introducing a new norm on $X$. Thus $T^{\odot *}(t)$ is an extension of $T(t)$ to the larger space $X^{\odot *}$. However, $T^{\odot *}(t)$ is not strongly continuous, but only weak $*$ continuous. If $f:[0, \tau] \rightarrow X^{\odot *}$ is a piecewise norm-continuous function (see Section 2 for a definition of piecewise continuity), then we can define the weak $*$ Riemann integral (see Section 2)

$$
F(t)=\int_{0}^{t} T^{\odot *}(t-s) f(s) \mathrm{d} s,
$$

and $F$ is a norm-continuous function on $[0, \tau]$ with values in $X$. (See proposition 2.4.) Now let us return to system (1.2). If the control $u(\bullet)$ is piecewise continuous, then the integral still makes sense if $B$ maps into $X^{\odot *}$ instead of $X$. So we allow $B$ to be a bounded linear operator from $X$ to $X^{\odot *}$, and replace (1.2) by

$$
x(t)=T(t) \phi+\int_{0}^{t} T^{\odot *}(t-s) B u(s) \mathrm{d} s .
$$

In this paper we concentrate on questions like controllability and observability, and it turns out that the results that we obtain greatly resemble those in the classical situation, where $B$ maps into $X$; see [7]. As an application, we discuss delay systems on the space of continuous functions.

In Section 2, we pursue the theory of dual $\mathrm{C}_{0}$-semigroups somewhat further. Among other things, we describe a perturbation result which we found in [2]. In Section 3 we use these results to construct a semigroup solution to a delay equation on the state space $\mathrm{C}[-r, 0]$. In Section 4 , we derive necessary and sufficient conditions for the approximate controllability of the system $(\Sigma)$. There we also show that controllability and observability are dual properties. In Section 5 , we reduce $(\Sigma)$ to a control system with a control operator $B$ mapping into $X$. We illustrate our abstract results with an application to the delay system of Section 3, to which a control term has been added. This is done in Section 6 . Finally, in Section 7, we make some remarks about the non-sun-reflexive case, about stabilizability, and about boundary control systems. 


\section{Dual semigroups, sun-reflexivity, and bounded perturbations}

In this section we describe some less-known results about dual $\mathrm{C}_{0}$-semigroups. Unless otherwise stated, proofs can be found in the book of Hille \& Phillips [9]; also see $[1,2]$. Let $X$ be a (real or complex) Banach space, and let $T(t)$ be a $\mathrm{C}_{0}$-semigroup on $X$ with (infinitesimal) generator $A$. Let $\omega_{0}(A)$ be the type of $T(t)$, i.e.

$$
\omega_{0}(A)=\inf _{t>0} \frac{1}{t} \log \|T(t)\|
$$

For every $\omega>\omega_{0}(A)$, there is a constant $M \geqslant 1$ such that

The right half plane

$$
\|T(t)\| \leqslant M \mathrm{e}^{\omega t} \quad(t \geqslant 0) .
$$

$$
D_{0}=\left\{\lambda \in \mathbb{C}: \operatorname{Re} \lambda>\omega_{0}(A)\right\},
$$

is entirely contained within the resolvent set $\rho(A)$ of $A$. Let $\langle\bullet \mid \bullet\rangle_{X}$ denote the pairing between elements of the space $X$ and its dual $X^{*}$. If there is no danger of confusion we omit the subscript $X$. We can embed $X$ in its bidual $X^{* *}$ via the canonical isometry $i: X \rightarrow X^{* *}$ given by

$$
\left\langle\phi^{*} \mid i(\phi)\right\rangle_{X^{*}}=\left\langle\phi \mid \phi^{*}\right\rangle_{X}, \quad \text { for every } \phi \in X \text { and } \phi^{*} \in X^{*} \text {. }
$$

We define the dual (or adjoint) semigroup $T^{*}(t)$ on $X^{*}$ by

$$
T^{*}(t)=T(t)^{*} \quad(t \geqslant 0) .
$$

Except for some special cases (e.g. if $X$ is reflexive or if $T(t)$ is uniformly continuous), $T^{*}(t)$ is not strongly continuous on the whole space $X^{*}$. Actually, $T^{*}(t)$ is a so-called weak * continuous semigroup on $X^{*}$, which means in particular that $t \mapsto\left\langle\phi \mid T^{*}(t) \phi^{*}\right\rangle_{X}$ is continuous, for every $\phi \in X$ and every $\phi^{*} \in X^{*}$. Let $A^{*}$ be the dual operator of $A$, which exists since $\mathfrak{P}(A)$ is dense in $X$.

THEOREM 2.1.

(a) $A^{*}$ is the weak * generator of $T^{*}(t)$, i.e. $\phi^{*}$ belongs to $\mathfrak{D}\left(A^{*}\right)$ if and only if $t^{-1}\left[T^{*}(t) \phi^{*}-\phi^{*}\right]$ converges as $t \downarrow 0$ with respect to the weak $*$ topology, and the limit equals $A^{*} \phi^{*}$ whenever there is convergence.

(b) If $\phi^{*} \in \mathfrak{D}\left(A^{*}\right)$, then $T^{*}(t) \phi^{*} \in \mathfrak{D}\left(A^{*}\right)$, for every $t \geqslant 0$, and $A^{*} T^{*}(t) \phi^{*}=$ $T^{*}(t) A^{*} \phi^{*}$.

Let $X^{\odot}$ be the subspace of $X^{*}$ on which $T^{*}(t)$ is strongly continuous, i.e.

$$
X^{\odot}=\left\{\phi^{*} \in X^{*}: \lim _{t \downarrow 0}\left\|T^{*}(t) \phi^{*}-\phi^{*}\right\|=0\right\} .
$$

It is easy to show that $X^{\odot}$ is a closed subspace of $X^{*}$ which is invariant under $T^{*}(t)$. Note that $X^{\odot}=X^{*}$, if $X$ is reflexive. We denote the restriction of $T^{*}(t)$ to $X^{\odot}$ by $T^{\odot}(t)$. It is obvious that $T^{\odot}(t)$ is a $\mathrm{C}_{0}$-semigroup on $X^{\odot}$. Let $A^{\odot}$ be its generator. 
THEOREM 2.2 .

(a) $X^{\odot}=$ clo $\mathfrak{D}\left(A^{*}\right)$.

(b) $A^{\odot}$ is the part of $A^{*}$ in $X^{\odot}$, i.e. $\phi^{\odot} \in \mathfrak{D}\left(A^{\odot}\right)$ if and only if $\phi^{\odot} \in \mathfrak{D}\left(A^{*}\right)$ and $A^{*} \phi^{\odot} \in X^{\odot}$. For such $\phi^{\odot}$, one has $A^{\odot} \phi^{\odot}=A^{*} \phi^{\odot}$.

(c) $\mathfrak{D}\left(A^{\odot}\right)$ is weak * dense in $X^{*}$.

Let the prime norm $\|\bullet\|^{\prime}$ on $X$ be defined by

$$
\|\phi\|^{\prime}=\sup \left\{\left|\left\langle\phi \mid \phi^{\odot}\right\rangle\right|: \phi^{\odot} \in X^{\odot},\left\|\phi^{\odot}\right\| \leqslant 1\right\} .
$$

This norm is equivalent to the original norm:

with

$$
\|\phi\|^{\prime} \leqslant\|\phi\| \leqslant M\|\phi\|^{\prime} \quad(\phi \in X),
$$

$$
M=\liminf _{\lambda \rightarrow \infty}\|\lambda R(\lambda, A)\|<\infty,
$$

where $R(\lambda, A)=(\lambda I-A)^{-1}$ is the resolvent operator. If $T(t)$ is a contraction semigroup, then the two norms coincide. If we equip $X$ with the prime norm, then this does not affect the norm on $X^{\odot}$, i.e.

$$
\left\|\phi^{\odot}\right\|=\sup \left\{\left|\left\langle\phi \mid \phi^{\odot}\right\rangle\right|: \phi \in X,\|\phi\|^{\prime} \leqslant 1\right\},
$$

for every $\phi^{\odot} \in X^{\odot}$.

We can repeat our game of taking duals and restrictions: $T^{\odot *}(t)$ is a weak $*$ continuous semigroup on $X^{\odot *}$ with weak $*$ generator $A^{\odot *}$. On $X^{\odot \odot}=$ clo $\Im\left(A^{\odot *}\right)$, the semigroup $T^{\odot *}(t)$ is strongly continuous, and $T^{\odot \odot}(t)$ denotes the restriction of $T^{\odot *}(t)$ to $X^{\odot \odot}$. Since every element of $X$ can be considered as a continuous linear functional on $X^{\odot}$, we can embed $X$ in $X^{\odot *}$ via the continuous injection $j: X \rightarrow X^{\odot *}$ defined by

$$
\left\langle\phi^{\odot} \mid j(\phi)\right\rangle_{X^{\odot}}=\left\langle\phi \mid \phi^{\odot}\right\rangle_{X}
$$

and it is clear that $j$ is an isometry if $X$ is equipped with the prime norm. A pleasant circumstance is that the prime norm on $X^{\odot \odot}$ is the same as the original norm. If $X$ has the prime norm, then $j$ maps $X$ isometrically into $X^{\odot \odot}$, and, in this sense, $T^{\odot \odot}(t)$ is an extension of $T(t)$ :

$$
T^{\odot \odot}(t) \circ j=j \circ T(t)
$$

From this point on, we shall identify $X$ with its embedding $j(X)$; this means in particular that we choose the prime norm on $X$.

In general, the space $X$ is smaller than $X^{\odot \odot}$. If they are the same, then we say that $X$ is $\odot$-reflexive with respect to $A$.

THEOREM 2.3.

(a) $X$ is $\odot$-reflexive with respect to $A$ if and only if $\mathrm{R}(\lambda, A)$ is $\sigma\left(X, X^{\odot}\right)$ compact.

(b) $X$ is $\odot$-reflexive with respect to $A$ if and only if $X^{\odot}$ is $\odot$-reflexive with respect to $A^{\odot}$.

In particular, Theorem 2.3a implies that $X$ is $\odot$-reflexive with respect to $A$ if the resolvent $R(\lambda, A)$ is compact. 
It is well-known that the solution of the inhomogeneous Cauchy problem

$$
\frac{\mathrm{d} x}{\mathrm{~d} t}(t)=A x(t)+f(t), \quad x(0)=\phi,
$$

where $f$ is an arbitrary norm-continuous $X$-valued function, is given by

$$
x(t)=T(t) \phi+\int_{0}^{t} T(t-s) f(s) \mathrm{d} s,
$$

where the integral can be interpreted as a Riemann integral. The result below shows that the integral is still meaningful for a much larger class of inhomogeneous terms $f$. Before we state this result, we describe the notion of a weak * Riemann integral.

Let $Z$ be a Banach space. Suppose that $h:[a, b] \rightarrow Z^{*}$ is a weak $*$ continuous function. Then, for every $\phi \in Z$, the Riemann integral

is well defined, and

$$
\int_{a}^{b}\langle\phi \mid h(t)\rangle \mathrm{d} t
$$

$$
\left|\int_{a}^{b}\langle\phi \mid h(t)\rangle \mathrm{d} t\right| \leqslant(b-a)\|\phi\| \sup _{0 \leqslant s \leqslant t}\|h(t)\| .
$$

Note that, by the uniform boundedness theorem, every weak * continuous function is (norm)-bounded. Thus

$$
\phi \mapsto \int_{a}^{b}\langle\phi \mid h(t)\rangle \mathrm{d} t
$$

defines a continuous linear functional on $Z$, and therefore corresponds to some element of $Z^{*}$, which we denote by

$$
\int_{a}^{b} h(t) \mathrm{d} t .
$$

We call this integral the weak $*$ Riemann integral of $h$.

Now, let us return to the integral in (2.3). If

is norm continuous, then

$$
f:[0, t] \rightarrow X^{\odot *}
$$

$$
s \mapsto T^{\odot *}(t-s) f(s)
$$

is a weak * continuous $X^{\odot *}$-valued function on $[0, t]$, and therefore we can define its weak * Riemann integral. This is still true if $f$ is only piecewise norm-continuous. A function on a bounded interval $I$ is called piecewise continuous, if it is continuous on $I$ except for at most a finite number of points, in which the left and right limits exist. For the proof of the following result we refer to [2].

Proposition 2.4 Let $f:[0, \tau] \rightarrow X^{\odot *}$ be a piecewise norm-continuous function, 
then

$$
t \mapsto \int_{0}^{t} T^{\odot *}(t-s) f(s) \mathrm{d} s
$$

is a norm-continuous $X^{\odot \odot}$-valued function on [0, $\left.\tau\right]$. If $T(t)$ obeys $(2.1)$ then

$$
\left\|\int_{0}^{t} T^{\odot *}(t-s) f(s) \mathrm{d} s\right\| \leqslant M \frac{\mathrm{e}^{\omega t}-1}{\omega} \sup _{0 \leqslant s \leqslant t}\|f(s)\| .
$$

In [2], Proposition 2.4 forms the basis of a rather useful perturbation theorem, which we describe below. We restrict ourselves to the following situation.

Let $T_{0}(t)$ be a $C_{0}$-semigroup on $X$ with infinitesimal generator $A_{0}$, and assume that $X$ is $\odot$-reflexive with respect to $A_{0}$. (In the final section we make some remarks about the non- $\odot$-reflexive case.) Consider a bounded linear perturbation

$$
C: X \rightarrow X^{\odot *} \text {. }
$$

Then $C^{*}$ maps $X^{\odot * *}$ into $X^{*}$, but here we are only interested in its restriction to $X^{\odot}$. The situation may be conveniently summarized by the following symmetric diagram:

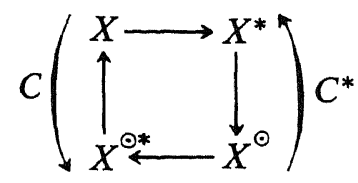

Here a horizontal arrow means 'taking the dual', and a vertical arrow 'taking the restriction'. We may construct the perturbed semigroup $T(t)$ on $X$ from the variation-of-constants formula

$$
T(t) \phi=T_{0}(t) \phi+\int_{0}^{t} T_{0}^{\odot *}(t-s) C T(s) \phi \mathrm{d} s,
$$

where the integral has to be interpreted as a weak $*$ Riemann integral. In [2], we show that indeed (2.4) defines a $\mathrm{C}_{0}$-semigroup $T(t)$. The spaces $X^{\odot}$ and $X^{\odot \odot}$ are the same for $T_{0}(t)$ and $T(t)$, which means in particular that $X$ is $\odot$-reflexive with respect to $A$, the generator of $T(t)$. This generator is given by

$$
\mathfrak{T}(A)=\left\{\phi \in \mathfrak{D}\left(A_{0}^{\odot *}\right): A_{0}^{\odot *} \phi+C \phi \in X\right\}, \quad A \phi=A_{0}^{\odot *} \phi+C \phi .
$$

For much more information on this perturbation result, we refer to [2]. We conclude this section with a technical lemma which is needed elsewhere in this paper. In this lemma and the sequel of this paper we adopt the following convention: by $\phi, \phi^{*}, \phi^{\odot}$, and $\phi^{\odot *}$ we denote arbitrary elements of $X, X^{*}, X^{\odot}$, and $X^{\odot *}$ respectively.

LeMMa 2.5 For every $\lambda \in D_{0}, \phi^{*} \in X^{*}$, and $\phi^{\odot *} \in X^{\odot *}$, we have
(a) $\left\langle\int_{0}^{t} \mathrm{e}^{-\lambda s} T^{\odot *}(s) \phi^{\odot *} \mathrm{~d} s \mid \phi^{*}\right\rangle_{X} \rightarrow\left\langle\mathrm{R}\left(\lambda, A^{\odot *}\right) \phi^{\odot *} \mid \phi^{*}\right\rangle_{X}$ as $t \rightarrow \infty$,
(b) $\left\langle\mathrm{R}\left(\lambda, A^{\odot *}\right) \phi^{\odot *} \mid \phi^{*}\right\rangle_{X}=\left\langle\mathrm{R}\left(\lambda, A^{*}\right) \phi^{*} \mid \phi^{\odot *}\right\rangle_{X^{\odot}}$. 
Proof. (a) Let $\mu \in D_{0}$; then

$$
\begin{gathered}
\left\langle\int_{0}^{t} \mathrm{e}^{-\lambda s} T^{\odot *}(s) \phi^{\odot *} \mathrm{~d} s \mid \mathrm{R}\left(\mu, A^{*}\right) \phi^{*}\right\rangle_{X}=\int_{0}^{t}\left\langle\mathrm{R}\left(\mu, A^{*}\right) \phi^{*} \mid \mathrm{e}^{-\lambda s} T^{\odot *}(s) \phi^{\odot *}\right\rangle_{X^{\odot}} \mathrm{d} s \\
=\int_{0}^{t}\left\langle\mathrm{e}^{-\lambda s} T^{\odot}(s) \mathrm{R}\left(\mu, A^{*}\right) \phi^{*} \mid \phi^{\odot *}\right\rangle_{X^{\odot}} \mathrm{d} s=\left\langle\int_{0}^{t} \mathrm{e}^{-\lambda s} T^{\odot}(s) \mathrm{R}\left(\mu, A^{*}\right) \phi^{*} \mathrm{~d} s \mid \phi^{\odot *}\right\rangle_{X^{\odot}} \\
=\left\langle\mathrm{R}\left(\lambda, A^{\odot}\right)\left[\mathrm{R}\left(\mu, A^{*}\right) \phi^{*}-\mathrm{e}^{-\lambda t} T^{\odot}(t) \mathrm{R}\left(\mu, A^{*}\right) \phi^{*}\right] \mid \phi^{\odot *}\right\rangle_{X^{\odot}} \\
=\left\langle\mathrm{R}\left(\lambda, A^{\odot *}\right) \phi^{\odot *} \mid \mathrm{R}\left(\mu, A^{*}\right) \phi^{*}-\mathrm{e}^{-\lambda t} T^{\odot}(t) \mathrm{R}\left(\mu, A^{*}\right) \phi^{*}\right\rangle_{X} \\
=\left\langle\mathrm{R}\left(\lambda, A^{\odot *}\right) \phi^{\odot *}-\mathrm{e}^{-\lambda t} T(t) \mathrm{R}\left(\lambda, A^{\odot *}\right) \phi^{\odot *} \mid \mathrm{R}\left(\mu, A^{*}\right) \phi^{*}\right\rangle_{X} .
\end{gathered}
$$

Here we have used that

$$
\int_{0}^{t} \mathrm{e}^{-\lambda s} T^{\odot}(s) \phi^{\odot} \mathrm{d} s=\mathrm{R}\left(\lambda, A^{\odot}\right)\left[\phi^{\odot}-\mathrm{e}^{-\lambda t} T^{\odot}(t) \phi^{\odot}\right] .
$$

Now, multiplying with $\mu$, and letting $\mu \rightarrow \infty$, we get

$$
\left\langle\int_{0}^{t} \mathrm{e}^{-\lambda s} T^{\odot *}(s) \phi^{\odot *} \mathrm{~d} s \mid \phi^{*}\right\rangle_{X}=\left\langle\mathrm{R}\left(\lambda, A^{\odot *}\right) \phi^{\odot *}-\mathrm{e}^{-\lambda t} T(t) \mathrm{R}\left(\lambda, A^{\odot *}\right) \phi^{\odot *} \mid \phi^{*}\right\rangle_{X} .
$$

From this, (a) follows immediately.

(b) As above, we get that

$$
\begin{aligned}
\left\langle\int_{0}^{t} \mathrm{e}^{-\lambda s}\right. & T^{\odot *}(s) \phi^{\odot *} \mathrm{~d} s\left|\phi^{*}\right\rangle_{X} \\
& =\left\langle\mathrm{R}\left(\lambda, A^{\odot}\right)\left[\mathrm{R}\left(\mu, A^{*}\right) \phi^{*}-\mathrm{e}^{-\lambda t} T^{\odot}(t) \mathrm{R}\left(\mu, A^{*}\right) \phi^{*}\right] \mid \phi^{\odot *}\right\rangle_{X^{\odot}} \\
& =\left\langle\mathrm{R}\left(\mu, A^{\odot}\right)\left[\mathrm{R}\left(\lambda, A^{*}\right) \phi^{*}-\mathrm{e}^{-\lambda t} T^{\odot}(t) \mathrm{R}\left(\lambda, A^{*}\right) \phi^{*}\right] \mid \phi^{\odot *}\right\rangle_{X^{\odot}}
\end{aligned}
$$

Multiplying with $\mu$, and letting $\mu \rightarrow \infty$ we get:

$$
\left\langle\int_{0}^{t} \mathrm{e}^{-\lambda s} T^{\odot *}(s) \phi^{\odot *} \mathrm{~d} s \mid \phi^{*}\right\rangle=\left\langle\mathrm{R}\left(\lambda, A^{*}\right) \phi^{*}-\mathrm{e}^{-\lambda t} T^{\odot}(t) \mathrm{R}\left(\lambda, A^{*}\right) \phi^{*} \mid \phi^{\odot *}\right\rangle_{X^{\odot}},
$$

and the expression at the right-hand-side converges to $\left\langle\mathrm{R}\left(\lambda, A^{*}\right) \phi^{*} \mid \phi^{\odot *}\right\rangle_{X^{\odot}}$ as $t \rightarrow \infty$. Now the result follows from (a).

\section{Delay equations}

In this section, which is based on a paper by Diekmann [5], we indicate how the perturbation theory for dual semigroups, described in the former section, can be used to construct semigroup solutions to delay equations on spaces of continuous functions.

Throughout this paper we assume that all bounded-variation functions on $[0, r]$ are normalized such that they are zero on $(-\infty, 0]$, right continuous on $(0, r)$, and constant on $[r, \infty)$. Let $H$ be a given $n \times n$ real-matrix function which is of bounded variation on $[0, r]$, and consider the retarded functional differential 
equation

$$
\dot{\boldsymbol{x}}(t)=\int_{0}^{r}[\mathrm{~d} H(\tau) \boldsymbol{x}(t-\tau)]
$$

with initial condition

$$
\boldsymbol{x}(\theta)=\varphi(\theta) \quad(-r \leqslant \theta \leqslant 0),
$$

where $\varphi \in X=\mathrm{C}\left([-r, 0] ; \mathbb{R}^{n}\right)$. In this section, we show how one can construct a semigroup $T(t)$ on $X$, associated with solutions of (3.1):

$$
[T(t) \varphi](\theta)=x_{t}(\theta ; \varphi) \quad(\theta \in[-r, 0]),
$$

where $x_{t}(\theta ; \varphi)=x(t+\theta ; \varphi)$, and $x(\bullet ; \varphi)$ is the solution of (3.1)-(3.2). As the unperturbed equation we take

$$
\dot{\boldsymbol{x}}(t)=\mathbf{0}
$$

considered as a retarded functional differential equation. The corresponding semigroup $T_{0}(t)$ on $X$ is given by

$$
\left[T_{0}(t) \phi\right](\theta)= \begin{cases}\phi(t+\theta) & \text { if } \theta+t \leqslant 0, \\ \phi(0) & \text { if } \theta+t \geqslant 0,\end{cases}
$$

which has generator $A_{0}$ given by

$$
A_{0} \phi=\phi^{\prime}, \quad \mathfrak{D}\left(A_{0}\right)=\left\{\phi \in C^{1}\left([-r, 0] ; \mathbb{R}^{n}\right): \phi^{\prime}(0)=0\right\} .
$$

If $X^{*}$ is represented by $\mathrm{BV}\left([0, r] ; \mathbb{R}^{n}\right)$, i.e. the space of bounded-variation $n$-vector-valued functions on $[0, r]$, with the pairing

$$
\langle\boldsymbol{\phi} \mid \boldsymbol{\psi}\rangle=\int_{0}^{r}\left[\mathrm{~d} \boldsymbol{\psi}(\tau)^{\top} \phi(-\tau)\right] \quad\left(\psi \in X^{*}, \phi \in X\right),
$$

where $T$ denotes transpose, then the dual semigroup $T_{0}^{*}(t)$ is given by

$$
\left[T_{0}^{*}(t) \psi\right](\theta)=\psi(t+\theta)
$$

Jne easily obtains that

$$
A_{0}^{\prime} \psi=\psi^{\prime}, \quad \mathfrak{D}\left(A_{0}^{*}\right)=\left\{\psi \in \mathbb{W}^{1,1}\left([0, r] ; \mathbb{R}^{n}\right): \psi^{\prime} \in X^{*}\right\} .
$$

Then $X^{\odot}=\operatorname{clo} \mathfrak{D}\left(A_{0}^{*}\right)=\mathrm{W}^{1,1}\left([0, r] ; \mathbb{R}^{n}\right)$. Every element $\psi \in \mathrm{W}^{1,1}\left([0, r] ; \mathbb{R}^{n}\right)$ corresponds to a pair $\left(\psi^{0}, \psi^{1}\right) \in M_{1}[0, r]:=\mathbb{R}^{n} \oplus \mathrm{L}^{1}\left([0, r] ; \mathbb{R}^{n}\right)$ in the sense that $\psi(\theta)=\psi^{0}+\int_{0}^{\theta} \psi^{1}(\tau) \mathrm{d} \tau(\theta \in[0, r])$. So we represent $X^{\odot}$ by $M_{1}[0, r]$. Obviously,

$$
T_{0}^{\odot}(t)\left(\boldsymbol{\psi}^{0}, \boldsymbol{\psi}^{1}\right)=\left(\boldsymbol{\psi}^{0}+\int_{0}^{t} \psi^{1}(\tau) \mathrm{d} \tau, \boldsymbol{\psi}_{t}^{1}\right)
$$

where, as before, $\psi_{t}^{1}(\theta)=\psi^{1}(t+\theta)$. Note that $T_{0}(t)$ is a contraction semigroup, so there is no need for changing norms. For $X^{\odot *}$, we choose the representation

$$
X^{\odot *}=M_{\infty}[-r, 0]=\mathbb{R}^{n} \oplus \mathrm{L}^{\infty}\left([-r, 0] ; \mathbb{R}^{n}\right)
$$


and we have the following pairing between $\left(\phi^{0}, \phi^{1}\right) \in M_{\infty}[-r, 0]$ and $\left(\psi^{0}, \psi^{1}\right) \in$ $M_{1}[0, r]$ :

$$
\left\langle\left(\psi^{0}, \psi^{1}\right) \mid\left(\phi^{0}, \phi^{1}\right)\right\rangle=\psi^{0 \top} \phi^{0}+\int_{0}^{r} \psi^{1}(\tau)^{\top} \phi^{1}(-\tau) \mathrm{d} \tau .
$$

A straightforward computation shows that

where

$$
T_{0}^{\odot *}(t)\left(\phi^{0}, \phi^{1}\right)=\left(\phi^{0}, \phi_{t}^{1}\right)
$$

$$
\phi_{t}^{1}(\theta)= \begin{cases}\phi^{1}(t+\theta) & \text { if } t+\theta \leqslant 0 \\ \phi^{0} & \text { if } t+\theta>0 .\end{cases}
$$

The generator $A_{0}^{\odot *}$ is given by

$$
\begin{gathered}
A_{0}^{\odot *}\left(\phi^{0}, \phi^{1}\right)=\left(0, \phi^{1 \prime}\right), \\
\mathfrak{D}\left(A_{0}^{\odot *}\right)=\left\{\left(\phi^{0}, \phi^{1}\right): \phi^{1} \in W^{1, \infty}\left([-r, 0] ; \mathbb{R}^{n}\right), \phi^{1}(0)=\phi^{0}\right\} .
\end{gathered}
$$

From $X^{\odot \odot}=$ clo $\mathfrak{D}\left(A_{0}^{\odot *}\right)$ we deduce that

$$
X^{\odot \odot}=\left\{\left(\phi^{0}, \phi^{1}\right) \in M_{\infty}[-r, 0]: \phi^{1} \in \mathrm{C}\left([-r, 0] ; \mathbb{R}^{n}\right), \phi^{1}(0)=\phi^{0}\right\},
$$

which is isomorphic with $X=\mathrm{C}\left([-r, 0] ; \mathbb{R}^{n}\right)$ in the sense that every element $\phi \in X$ corresponds to a unique element $(\phi(0), \phi) \in X^{\odot \odot}$. In the sequel, we shall identify the two spaces. Notice that the sun-reflexivity also follows from the compactness of $R\left(\lambda, A_{0}\right)$. As we noted, the unperturbed equation (3.4) corresponds to the abstract Cauchy problem

$$
\frac{\mathrm{d}}{\mathrm{d} t} \boldsymbol{x}_{t}=A_{0} x_{t}, \quad x_{0}=\varphi
$$

Let $C: X \rightarrow X^{\odot *}$ be the bounded linear operator given by

$$
C \phi=\left(\int_{0}^{r}[\mathrm{~d} H(\tau) \phi(-\tau)], \mathbf{0}\right) .
$$

The perturbation results of the former section apply, and we deduce from (2.5) that $A$ given by

$$
A \phi=\phi^{\prime}, \quad \mathfrak{S}(A)=\left\{\phi \in C^{1}\left([-r, 0] ; \mathbb{R}^{n}\right): \phi^{\prime}(0)=\int_{0}^{r}[\mathrm{~d} H(\tau) \phi(-\tau)]\right\},
$$

is the generator of a $\mathrm{C}_{0}$-semigroup $T(t)$, which is actually the semigroup given by (3.3).

In [5], Diekmann elaborates this example further, exploiting the fact that the range of the perturbation operator $C$ is finite-dimensional. He also indicates that, if one starts from the renewal equation

$$
z=\psi+H^{\top} \star z
$$

where $\psi \in X^{\odot}$, then $T^{\odot}(t) \psi$ is given by

$$
z_{t}=T^{\odot}(t) \psi+H^{\top} \star z_{t} .
$$




\section{Controllability and observability}

Let $X$ be a (real or complex) Banach space, and let $T(t)$ be a $\mathrm{C}_{0}$-semigroup on $X$ with generator $A$. Throughout this and the next section we assume the following.

BASIC Assumption: $X$ is $\odot$-reflexive with respect to $A$.

Let $U$ be another Banach space (the control space) and assume that

$$
B: U \rightarrow X^{\odot *}
$$

is a bounded linear operator. By $\mathscr{C}_{\tau}(U)$, or just $\mathscr{C}_{\tau}$, we denote the space of piecewise continuous functions $u:[0, \tau] \rightarrow U$ (see Section 2 for a definition). We consider the control system on $X$ given by

$$
x(t)=T(t) \phi+\int_{0}^{t} T^{\odot *}(t-s) B u(s) \mathrm{d} s .
$$

Here the integral should be interpreted as a weak $*$ Riemann integral; see Section 2. Now Proposition 2.4 implies that $x(\bullet)$ defines a continuous $X$-valued function on $[0, \tau]$ if $u \in \mathscr{C}_{\tau}$. Define the linear subspace $\Omega_{t}$ of $X$ by

$$
\Omega_{t}=\left\{\int_{0}^{t} T^{\odot *}(t-s) B u(s) \mathrm{d} s: u \in \mathscr{C}_{t}\right\} .
$$

We also define

$$
\Omega=\bigcup_{t>0} \Omega_{t}
$$

$\Omega$ is the set containing all points of $X$ to which the origin can be steered in finite time, and it is called the controllability space.

Definttion We say that the control system $(\Sigma)$ is approximately controllable if $\Omega$ is dense in $X$.

In the case that $X$ has finite dimension, there exists a simple criterion for controllability in terms of $A$ and $B$; see Curtain \& Pritchard [3]. Below, we shall formulate a generalization of this criterion in terms of the resolvent $R(\lambda, A)$ and $B$; see Theorem 4.6. We start with some preparatory results.

LEMMA 4.1 If $\phi \in \Omega_{t}$, then $T(h) \phi \in \Omega_{t+h}$.

Proof. Let $\phi \in \Omega_{t}$, then $\phi=\int_{0}^{t} T^{\odot *}(t-s) B u(s) \mathrm{d} s$ for some $u \in \mathscr{C}_{t}$. Define $u_{h} \in \mathscr{C}_{t+h}$ by

$$
u_{h}(s)= \begin{cases}u(s) & \text { if } 0 \leqslant s \leqslant t \\ 0 & \text { if } t<s \leqslant t+h\end{cases}
$$

Then

$$
T(h) \phi=\int_{0}^{t} T^{\odot *}(t+h-s) B u(s) \mathrm{d} s=\int_{0}^{t+h} T^{\odot *}(t+h-s) B u_{h}(s) \mathrm{d} s \in \Omega_{t+h} .
$$


A direct consequence of this result is

Proposition $4.2 \Omega$ is invariant under $T(t)$.

It is clear that system $(\Sigma)$ is approximately controllable if and only if $\phi^{*} \in X^{*}$, with $\phi^{*} \perp \Omega$, implies that $\phi^{*}=0$. Here $\phi^{*} \perp \Omega$ means that $\left\langle\phi \mid \phi^{*}\right\rangle=0$ for every $\phi \in \Omega$. Let $\omega_{0}(A)$ be the type of the semigroup $T(t)$ and let $D_{0}$ be the right half plane given by $(2.2)$, i.e.

$$
D_{0}=\left\{\lambda \in \mathbb{C}: \operatorname{Re} \lambda>\omega_{0}(A)\right\} .
$$

Proposition $4.3 \phi^{*} \perp \Omega$ if and only if $\mathrm{R}\left(\lambda, A^{*}\right) \phi^{*} \perp \Omega$ for every $\lambda \in D_{0}$.

Proof. 'only if': Suppose $\phi^{*} \perp \Omega$. Choose $\phi \in \Omega$. From Proposition 4.2 we infer that $\left\langle T(s) \phi, \phi^{*}\right\rangle=0$, for every $s \geqslant 0$. But this yields that $\int_{0}^{t}\left\langle\mathrm{e}^{-\lambda s} T(s) \phi \mid \phi^{*}\right\rangle \mathrm{d} s=0$, for every $t \geqslant 0$ and $\lambda \in \mathbb{C}$. Take $\lambda \in D_{0}$ and let $t \rightarrow \infty$. We get that

$$
\left\langle\mathrm{R}(\lambda, A) \phi \mid \phi^{*}\right\rangle=\left\langle\phi \mid \mathrm{R}\left(\lambda, A^{*}\right) \phi^{*}\right\rangle=0 .
$$

This holds for arbitrary $\phi \in \Omega$, which proves that $\mathrm{R}\left(\lambda, A^{*}\right) \phi^{*} \perp \Omega$.

'if': Now suppose that $\mathrm{R}\left(\lambda, A^{*}\right) \phi^{*} \perp \Omega$ for every $\phi \in D_{0}$. Then $\left\langle\phi \mid R\left(\lambda, A^{*}\right) \phi^{*}\right\rangle=0$ for every $\phi \in \Omega$. If we multiply with $\lambda$, and let $\lambda \rightarrow \infty$ we get that $\left\langle\phi \mid \phi^{*}\right\rangle=0$, which yields the result.

Proposition $4.4 \phi^{*} \perp \Omega$ if and only if $B^{*} \mathrm{R}\left(\lambda, A^{*}\right)^{k} \phi^{*}=0$ for every $\lambda \in D_{0}$ and $k=1,2, \ldots$

Proof. 'only if': Assume that $\phi^{*} \perp \Omega$. We first show that $B^{*} \mathrm{R}\left(\lambda, A^{*}\right) \phi^{*}=0$ for every $\lambda \in D_{0}$. Then the 'only if' part follows with Proposition 4.3. By definition,

$$
\left\langle\int_{0}^{t} T^{\odot *}(t-s) B u(s) \mathrm{d} s \mid \phi^{*}\right\rangle=0,
$$

for every $t \geqslant 0$ and $u \in \mathscr{C}_{t}$. Let $\lambda \in D_{0}$ and $u \in U$, and substitute $u(s)=\mathrm{e}^{\lambda s} u$. This yields that

$$
\left\langle\int_{0}^{\mathrm{t}} \mathrm{e}^{-\lambda s} T^{\odot *}(s) B u \mathrm{~d} s \mid \phi^{*}\right\rangle=0 .
$$

Letting $t \rightarrow \infty$, and using Lemma $2.5 \mathrm{a}$, we find that $\left\langle\mathrm{R}\left(\lambda, A^{\odot *}\right) B u \mid \phi^{*}\right\rangle=0$. With Lemma $2.5 \mathrm{~b}$, this can be rewritten as $\left\langle u \mid B^{*} \mathrm{R}\left(\lambda, A^{*}\right) \phi^{*}\right\rangle_{U}=0$. But this must hold for every $u \in U$, and we infer that $B^{*} \mathrm{R}\left(\lambda, A^{*}\right) \phi^{*}=0$.

'if': Assume that $B^{*} \mathrm{R}\left(\lambda, A^{*}\right)^{k} \phi^{*}=0$ for every $\lambda \in D_{0}$ and $k=1,2, \ldots$ Let $\lambda_{0} \in D_{0}$ be fixed. From the resolvent equation

we get that

$$
\mathrm{R}\left(\lambda, A^{*}\right)-\mathrm{R}\left(\lambda_{0}, A^{*}\right)=\left(\lambda_{0}-\lambda\right) \mathrm{R}\left(\lambda, A^{*}\right) \mathrm{R}\left(\lambda_{0}, A^{*}\right),
$$

$B^{*} \mathrm{R}\left(\lambda, A^{*}\right)^{k} \mathrm{R}\left(\lambda_{0}, A^{*}\right) \phi^{*}=\frac{1}{\lambda_{0}-\lambda} B^{*} \mathrm{R}\left(\lambda, A^{*}\right)^{k-1}\left[\mathrm{R}\left(\lambda, A^{*}\right) \phi^{*}-\mathrm{R}\left(\lambda_{0}, A^{*}\right) \phi^{*}\right]=0$

if we assume that $B^{*} \mathrm{R}\left(\lambda, A^{*}\right)^{k-1} \mathrm{R}\left(\lambda_{0}, A^{*}\right) \phi^{*}=0$. Thus, by induction, we get that

$$
B^{*} \mathrm{R}\left(\lambda, A^{*}\right)^{k} \mathrm{R}\left(\lambda_{0}, A^{*}\right) \phi^{*}=0,
$$


for every $\lambda \in D_{0}$ and $k=0,1,2, \ldots$ Since $\mathrm{R}\left(\lambda_{0}, A^{*}\right)$ maps $X^{*}$ into $\mathfrak{S}\left(A^{*}\right) \subset X^{\odot}$, we may write

$$
B^{*} \mathrm{R}\left(\lambda, A^{\odot}\right)^{k} \mathrm{R}\left(\lambda_{0}, A^{*}\right) \phi^{*}=0 .
$$

Now, let $y>0$ be fixed and choose $\lambda=k / t$, where $k$ is so large that $k / t \in D_{0}$. Then

$$
B^{*}(k / t)^{k} \mathrm{R}\left(k / t, A^{\odot}\right)^{k} \mathrm{R}\left(\lambda_{0}, A^{*}\right) \phi^{*}=0 .
$$

We let $k \rightarrow \infty$ and find that

$$
B^{*} T^{\odot}(t) \mathrm{R}\left(\lambda_{0}, A^{*}\right) \phi^{*}=0 .
$$

Let $\phi \in \Omega, \phi=\int_{0}^{t} T^{\odot *}(t-s) B u(s) \mathrm{d} s$, for some $t>0$, and $u \in \mathscr{C}_{t}$. Then

$$
\begin{aligned}
\left\langle\phi \mid \mathrm{R}\left(\lambda_{0}, A^{*}\right) \phi^{*}\right\rangle & =\int_{0}^{t}\left\langle\mathrm{R}\left(\lambda_{0}, A^{*}\right) \phi^{*} \mid T^{\odot *}(t-s) B u(s)\right\rangle_{X^{\odot}} \mathrm{d} s \\
& =\int_{0}^{t}\left\langle u(s) \mid B^{*} T^{\odot}(t-s) \mathrm{R}\left(\lambda_{0}, A^{*}\right) \phi^{*}\right\rangle_{U} \mathrm{~d} s=0 .
\end{aligned}
$$

Thus $\mathrm{R}\left(\lambda_{0}, A^{*}\right) \phi^{*} \perp \Omega$, and Proposition 4.3 implies that $\phi^{*} \perp \Omega$.

On the right-half-plane $D_{0}$, the resolvent $\mathrm{R}\left(\lambda, A^{*}\right)$ is an analytic function, and

$$
\frac{\mathrm{d}^{k}}{\mathrm{~d} \lambda^{k}} \mathrm{R}\left(\lambda, A^{*}\right)=(-1)^{k} k ! \mathrm{R}\left(\lambda, A^{*}\right)^{k+1} .
$$

This important fact forms the basis for the proof of our next result.

THEOREM 4.5 The following assertions are equivalent.

(a) $\phi^{*} \perp \Omega$

(b) $B^{*} \mathrm{R}\left(\lambda, A^{*}\right) \phi^{*}=0$ for every $\lambda \in D_{0}$

(c) $B^{*} \mathrm{R}\left(\lambda_{0}, A^{*}\right)^{k} \phi^{*}=0$ for some $\lambda_{0} \in D_{0}$ and every $k=1,2, \ldots$

Proof. (a) $\Rightarrow$ (b): follows from Proposition 4.4 .

(b) $\Rightarrow$ (a): if $B^{*} R\left(\lambda, A^{*}\right) \phi^{*}=0$ for every $\lambda \in D_{0}$, then

$$
\frac{\mathrm{d}^{k}}{\mathrm{~d} \lambda^{k}}\left[B^{*} \mathrm{R}\left(\lambda, A^{*}\right) \phi^{*}\right]=0
$$

for every $\lambda \in D_{0}$ and $k=0,1,2, \ldots$ Now (a) follows from (4.3) and Proposition 4.4.

(a) $\Rightarrow$ (c): follows from Proposition 4.4 .

(c) $\Rightarrow$ (b):

$$
\mathrm{R}\left(\lambda, A^{*}\right)=\sum_{k=0}^{\infty} \frac{1}{k !}\left(\lambda-\lambda_{0}\right)^{k}\left[\frac{\mathrm{d}^{k}}{\mathrm{~d} \lambda^{k}} \mathrm{R}\left(\lambda, A^{*}\right)\right]_{\lambda=\lambda_{0}}=\sum_{k=0}^{\infty}\left(\lambda_{0}-\lambda\right)^{k} \mathrm{R}\left(\lambda_{0}, A^{*}\right)^{k+1}
$$

for every $\lambda$ in a sufficiently small neighbourhood $\mathcal{O}$ of $\lambda_{0}$. Thus (c) yields that $B^{*} R\left(\lambda, A^{*}\right) \phi^{*}=0$ for every $\lambda \in \mathcal{O}$. But now the analyticity of $\mathrm{R}\left(\lambda, A^{*}\right)$ implies that this holds for every $\lambda \in D_{0}$. 
We are now ready to prove our main result.

THEOREM 4.6 The following assertions are equivalent.

(a) System $(\Sigma)$ is approximately controllable

(b) $B^{*} \mathrm{R}\left(\lambda, A^{*}\right) \phi^{*}=0$ for every $\lambda \in D_{0}$ implies $\phi^{*}=0$

(c) $B^{*} R\left(\lambda, A^{\odot}\right) \phi^{\odot}=0$ for every $\lambda \in D_{0}$ implies $\phi^{\odot}=0$

(d) clo $\operatorname{span}\left\{\mathrm{R}\left(\lambda, A^{\odot *}\right) B u: u \in U, \lambda \in D_{0}\right\}=X$

(e) $\phi^{\odot} \perp$ clo span $\left\{\mathrm{R}\left(\lambda, A^{\odot *}\right) B u: u \in U, \lambda \in D_{0}\right\}$ implies $\phi^{\odot}=0$.

Proof. (a) $\Leftrightarrow$ (b) by Theorem 4.5 .

(b) $\Rightarrow$ (c): trivial.

(c) $\Rightarrow$ (b): assume (c). Fix $\lambda_{0} \in D_{0}$. If $B^{*} R\left(\lambda, A^{*}\right) \phi^{*}=0$ for every $\lambda \in D_{0}$, then

$$
B^{*} \mathrm{R}\left(\lambda, A^{\odot}\right) \mathrm{R}\left(\lambda_{0}, A^{*}\right) \phi^{*}=0,
$$

for every $\lambda \in D_{0}$. This follows from the resolvent equation (4.2). From (c) we conclude that $\mathrm{R}\left(\lambda_{0}, A^{*}\right) \phi^{*}=0$, thus $\phi^{*}=0$. This proves (b).

(b) $\Rightarrow$ (d): assume (b). Suppose that

Thus

$$
\phi^{*} \perp \text { clo span }\left\{\mathrm{R}\left(\lambda, A^{\odot *}\right) B u: u \in U, \lambda \in D_{0}\right\} .
$$

$$
\left\langle\mathrm{R}\left(\lambda, A^{\odot *}\right) B u \mid \phi^{*}\right\rangle=0
$$

for every $\lambda \in D_{0}$ and $u \in U$. From Lemma $2.5 \mathrm{~b}$ we infer that $\left\langle u \mid B^{*} \mathrm{R}\left(\lambda, A^{*}\right) \phi^{*}\right\rangle=0$ for every $u \in U$ and $\lambda \in D_{0}$. Thus $B^{*} \mathrm{R}\left(\lambda, A^{*}\right) \phi^{*}=0$ for every $\lambda \in D_{0}$. Now (b) yields that $\phi^{*}=0$.

(d) $\Rightarrow$ (e): trivial.

(e) $\Rightarrow$ (c): straightforward.

Our next issue is observability. Let $V$ be a Banach space and $F: X \rightarrow V$ a bounded linear operator. Let $x(\bullet)$ be determined by system $(\Sigma)$, and suppose that one is able to measure $v(t)=F x(t)$, which we call the output vector. An important problem in control theory is whether the initial state $\varphi$ can be determined uniquely if the control (= input) $u(\bullet)$ and the output $v(\bullet)$ are given. Consider two initial states $\varphi_{1}$ and $\varphi_{2}$, and let $x_{1}\left(\bullet^{\bullet}\right)$ and $x_{2}(\bullet)$ be the corresponding solutions of $(\Sigma)$, both for the same control $u(\bullet)$. If $v_{1}(t)=v_{2}(t)$, that is, $F x_{1}(t)=F x_{2}(t)$, then

$$
F T(t)\left(\varphi_{1}-\varphi_{2}\right)=0 \text {. }
$$

Definition The control system $(\Sigma)$ is said to be observable by $F$ if $F T(t) \phi=0$ $(t \geqslant 0)$ implies that $\phi=0$.

Since the observability of $(\Sigma)$ does not depend on the control operator $B$, one might also say that the semigroup $T(t)$ is observable by $F$. The theorem below shows that controllability and observability are dual properties. As before, we consider $B^{*}$ as a bounded operator from $X^{\odot}$ to $U^{*}$.

THEOREM 4.7 The system $(\Sigma)$ is approximately controllable if and only if $T^{\odot}(t)$ is observable by $B^{*}$.

Proof. 'only if': Assume that $(\Sigma)$ is approximately controllable, and that 
$B^{*} T^{\odot}(t) \phi^{\odot}=0$ for $t \geqslant 0$. Taking the Laplace transform we get that $B^{*} \mathrm{R}\left(\lambda, A^{\odot}\right) \phi^{\odot}=0$ for every $\lambda \in D_{0}$. But now, Theorem 4.6 implies that $\phi^{\odot}=0$.

'if': Assume that $T^{\odot}(t)$ is observable by $B^{*}$, and that $B^{*} \mathrm{R}\left(\lambda, A^{\odot}\right) \phi^{\odot}=0$ for every $\lambda \in D_{0}$. Choose $\lambda=k / t$, where $k$ is so large that $k / t \in D_{0}$. Then

$$
B^{*}(k / t)^{k} \mathrm{R}\left(k / t, A^{\odot}\right)^{k} \phi^{\odot}=0 .
$$

Letting $k \rightarrow \infty$, we get $B^{*} T^{\odot}(t) \phi^{\odot}=0(t \geqslant 0)$. Since $T^{\odot}(t)$ is observable by $B^{*}$ we infer that $\phi^{\odot}=0$, and Theorem 4.6 implies that $(\Sigma)$ is approximately controllable.

\section{A transformed control system}

In this section, we show that system $(\Sigma)$ can be 'reduced' to another system in which the control operator $B$ maps $U$ into $X$. We start with a lemma.

LEMMA 5.1 Let $f:[0, t] \rightarrow X^{\odot *}$ be piecewise norm-continuous, and let $\lambda \in D_{0}$. Then

$$
\int_{0}^{t} T(t-s) \mathrm{R}\left(\lambda, A^{\odot *}\right) f(s) \mathrm{d} s=\mathrm{R}(\lambda, A) \int_{0}^{t} T^{\odot *}(t-s) f(s) \mathrm{d} s .
$$

Proof. Let $\phi^{\odot} \in X^{\odot}$ be arbitrary. Then

$$
\begin{aligned}
&\left\langle\int_{0}^{t} T(t-s) \mathrm{R}\left(\lambda, A^{\odot *}\right) f(s) \mathrm{d} s \mid \phi^{\odot}\right\rangle_{X}=\int_{0}^{t}\left\langle\mathrm{R}\left(\lambda, A^{\odot}\right) T^{\odot}(t-s) \phi^{\odot} \mid f(s)\right\rangle_{X^{\odot} \mathrm{d} s} \\
&=\int_{0}^{t}\left\langle T^{\odot}(t-s) \mathrm{R}\left(\lambda, A^{\odot}\right) \phi^{\odot} \mid f(s)\right\rangle_{X^{\odot} \mathrm{d} s}=\left\langle\int_{0}^{t} T^{\odot *}(t-s) f(s) \mathrm{d} s \mid \mathrm{R}\left(\lambda, A^{\odot}\right) \phi^{\odot}\right\rangle_{X} \\
&=\left\langle\mathrm{R}(\lambda, A) \int_{0}^{t} T^{\odot *}(t-s) f(s) \mathrm{d} s \mid \phi^{\odot}\right\rangle_{X}
\end{aligned}
$$

But $X^{\odot}$ lies weak * dense in $X^{*}$, and the proof is complete.

Let $\lambda \in D_{0}$. Applying $R(\lambda, A)$ to $(\Sigma)$, we get from Lemma 5.1:

$$
\hat{x}(t)=T(t) \hat{\phi}+\int_{0}^{t} T(t-s) \hat{B}_{\lambda} u(s) \mathrm{d} s,
$$

where $\hat{\phi}=\mathrm{R}(\lambda, A) \phi \in \mathfrak{D}(A)$, and $\hat{B}_{\lambda}=\mathrm{R}\left(\lambda, A^{\odot *}\right) B$, which is a bounded linear operator from $U$ into $X$. Notice that $\hat{x}(t)$, given by $\left(\hat{\Sigma}_{\lambda}\right)$, defines a classical (= continuously differentiable) solution of the inhomogeneous differential equation

$$
\frac{\mathrm{d} \hat{x}}{\mathrm{~d} t}(t)=A \hat{x}(t)+\hat{B}_{\lambda} u(t), \quad \hat{x}(0)=\hat{\phi},
$$

if $\hat{\phi} \in \mathfrak{S}(A)$ and $u(\bullet)$ is continuously differentiable.

The controllability space $\hat{\Omega}_{\lambda}$ corresponding with $\left(\hat{\Sigma}_{\lambda}\right)$ is given by

$$
\hat{\Omega}_{\lambda}=\bigcup_{t>0} \hat{\Omega}_{\lambda, t}
$$

where $\hat{\Omega}_{\lambda, t}=\left\{\int_{0}^{t} T(t-s) \hat{B}_{\lambda} u(s) \mathrm{d} s: u \in \mathscr{C}_{t}\right\}$. 
In the rest of this section, we describe how approximate controllability of $(\Sigma)$ and $\left(\hat{\Sigma}_{\lambda}\right)$ are related. From Lemma 5.1 we infer that

$$
\int_{0}^{t} T(t-s) \hat{B}_{\lambda} u(s) \mathrm{d} s=\mathrm{R}(\lambda, A) \int_{0}^{t} T^{\odot *}(t-s) B u(s) \mathrm{d} s,
$$

for every $u \in \mathscr{C}_{t}$, which yields that $\hat{\Omega}_{\lambda, t}=\mathrm{R}(\lambda, A) \Omega_{t}(t>0)$ and therefore

$$
\hat{\Omega}_{\lambda}=\mathrm{R}(\lambda, A) \Omega \text {. }
$$

Proposition 5.2 clo $\hat{\Omega}_{\lambda}=$ clo $\Omega$, for every $\lambda \in D_{0}$.

Proof. Let $\lambda \in D_{0}$. We show that $\phi^{*} \perp \hat{\Omega}_{\lambda}$ if and only if $\phi^{*} \perp \Omega$.

'only if': assume that $\phi^{*} \perp \hat{\Omega}_{\lambda}$. Because of (5.1), this is equivalent to

$$
\mathrm{R}\left(\lambda, A^{*}\right) \phi^{*} \perp \Omega \text {. }
$$

Let $\mu \in D_{0}$ be arbitrary. From Proposition 4.3 we infer that

$$
\mathrm{R}\left(\mu, A^{*}\right) \mathrm{R}\left(\lambda, A^{*}\right) \phi^{*} \perp \Omega .
$$

Now the resolvent equation (4.2) yields that $\mathrm{R}\left(\mu, A^{*}\right) \phi^{*} \perp \Omega$. Since $\mu$ was arbitrary, we may apply Proposition 4.3 once more, from which we get that $\phi^{*} \perp \Omega$.

'if': follows from Proposition 4.3.

The main conclusion of this section is

COROLlaRy $5.3(\Sigma)$ is approximately controllable if and only if $\left(\hat{\Sigma}_{\lambda}\right)$ is approximately controllable, for some $\lambda \in D_{0}$.

\section{An application: controllability of delay equations}

In Section 3, we demonstrated the usefulness of the theory of dual $\mathrm{C}_{0^{-}}$ semigroups on nonreflexive Banach spaces with respect to the study of delay equations. In the present section, we consider the delay equation (3.1) with an extra control term:

$$
\dot{\boldsymbol{x}}(t)=\int_{0}^{r}[\mathrm{~d} H(\tau) \boldsymbol{x}(t-\tau)]+B \boldsymbol{u}(t),
$$

with initial condition

$$
\boldsymbol{x}(\theta)=\varphi(\theta) \quad(\theta \in[-r, 0])
$$

where $\boldsymbol{\varphi}$ and $H$ are as in Section $3, \boldsymbol{u}(t)$ is a piecewise continuous $\mathbb{R}^{m}$-valued function, and $B$ is a real $n \times m$-matrix. Below, we shall indicate how the system (6.1)-(6.2) can be written as an abstract control system $(\Sigma)$; see Section 4. Let $X$, $X^{*}, X^{\odot}, X^{\odot *}, T(t), A$, and $\mathfrak{D}(A)$ be as in Section 3. Define $U=\mathbb{R}^{m}$, and let $B: U \rightarrow X^{\odot *}$ be the bounded linear operator given by

$$
B u=(B u, 0) \text {. }
$$

Now (6.1)-(6.2) can be written abstractly as

$$
\boldsymbol{x}_{t}=T(t) \varphi+\int_{0}^{t} T^{\odot *}(t-s) B \boldsymbol{u}(s) \mathrm{d} s,
$$


which is of the form $(\Sigma)$. We shall now state conditions which guarantee approximate controllability of (6.4). In terms of the original system (6.1)-(6.2), this is called function-space controllability: see $[10,11,13]$. We use Theorem 4.6: to be precise, the equivalence of (a) and (c). First we compute $R\left(\lambda, A^{\odot *}\right)$. Since $\mathrm{R}\left(\lambda, A^{\odot *}\right)$ maps $X^{\odot *}$ into $\mathfrak{D}\left(A^{\odot *}\right) \subset X$, the equation

reduces to

$$
\lambda\left(\phi^{0}, \phi^{1}\right)-A^{\odot *}\left(\phi^{0}, \phi^{1}\right)=\left(f^{0}, f^{1}\right),
$$

$$
\lambda \varphi(0)-\int_{0}^{r}[\mathrm{~d} H(\tau) \varphi(-\tau)]=f^{0}, \quad \lambda \varphi-\varphi^{\prime}=f^{1} .
$$

An easy computation shows that this system is equivalent to

$$
\begin{gathered}
\Delta(\lambda) \varphi(0)=f^{0}+\int_{0}^{r}\left(\mathrm{~d} H(\tau) \int_{-\tau}^{0} f^{1}(\sigma) \mathrm{e}^{\lambda(\sigma+\tau)} \mathrm{d} \sigma\right), \\
\varphi(\theta)=\mathrm{e}^{\lambda \theta} \varphi(0)+\int_{\theta}^{0} f^{1}(\sigma) \mathrm{e}^{\lambda(\theta-\sigma)} \mathrm{d} \sigma .
\end{gathered}
$$

Here the $n \times n$-matrix $\Delta(\lambda)$ is given by

$$
\Delta(\lambda)=\lambda I-\int_{0}^{r} \mathrm{e}^{-\lambda \tau} \mathrm{d} H(\tau) .
$$

The spectrum of $A$, as well as the spectrum of $A^{\odot *}$, consists entirely of eigenvalues $\lambda$, which are solutions of the characteristic equation

$$
\operatorname{det} \Delta(\lambda)=0 \text {. }
$$

If $\lambda \in \rho(A)=\rho\left(A^{\odot *}\right)$, where $\rho(\bullet)$ denotes the resolvent set, then

$$
\begin{aligned}
& \mathrm{R}\left(\lambda, A^{\odot *}\right)\left(f^{0}, f^{1}\right)= \\
& \mathrm{e}^{\lambda \theta} \Delta(\lambda)^{-1}\left[f^{0}+\int_{0}^{r}\left(\mathrm{~d} H(\tau) \int_{-\tau}^{0} \boldsymbol{f}^{1}(\sigma) \mathrm{e}^{-\lambda(\sigma+\tau)} \mathrm{d} \sigma\right)\right]+\int_{\theta}^{0} f^{1}(\sigma) \mathrm{e}^{\lambda(\theta-\sigma)} \mathrm{d} \sigma .
\end{aligned}
$$

It follows that, for $\boldsymbol{u} \in U=\mathbb{R}^{m}$,

$$
\left[\mathrm{R}\left(\lambda, A^{\odot *}\right) B u\right](\theta)=\mathrm{e}^{\lambda \theta} \Delta(\lambda)^{-1} B u .
$$

The following result is implied by Theorem 4.6.

THEOREM 6.1 System (6.4) is approximately controllable if and only if $\left(\psi^{0}, \psi^{1}\right) \in$ $M_{1}[0, r]$, with

$$
\left(\psi^{0}+\int_{0}^{r} \psi^{1}(\theta) \mathrm{e}^{-\lambda \theta} \mathrm{d} \theta\right)^{\top} \Delta(\lambda)^{-1} B u=0
$$

for every $\lambda$ satisfying $\operatorname{Re} \lambda>\omega_{0}(A)$ and for every $u \in U$, implies that $\psi^{0}=\psi^{1}=0$.

In particular, this theorem guarantees approximate controllability if rank $B=n$. In [11], Manitius \& Triggiani study delay equations and their approximate controllability on the Hilbert space $M_{2}[-r, 0]=\mathbb{R}^{n} \oplus \mathrm{L}^{2}\left([-r, 0] ; \mathbb{R}^{n}\right)$. The (necessary and sufficient) condition that they find is very similar to our Theorem 
6.1 , the only difference being that the condition $\left(\psi^{0}, \psi^{1}\right) \in M_{1}[0, r]$ has to be replaced by $\left(\psi^{0}, \psi^{1}\right) \in M_{2}[0, r]$. Actually, Manitius \& Triggiani pursue the problem much further, and use results of Hardy on entire functions and the Paley-Wiener theorem to derive conditions on $H$ and $B$ which guarantee approximate controllability. It is likely that their results can be extended to the present situation. See also [10].

\section{Final remarks}

In Section 4 we made the basic assumption that $X$ is sun-reflexive with respect to $A$. What can be said if this assumption is not satisfied? The answer to this question is that, in essence, most of the results remain valid, at least if one is disposed to choose, instead of $X$, the larger space $X^{\odot \odot}$ as the underlying state space. In [2, in prep.], we show that one can define a bilinear continuous mapping $[\bullet \mid \bullet]: X^{\odot \odot} \times X^{*} \rightarrow \mathbb{C}$ as follows

$$
\left[\phi^{\odot \odot} \mid \phi^{*}\right]=\lim _{t \downarrow 0}\left\langle\frac{1}{t} \int_{0}^{t} T^{*}(s) \phi^{*} \mathrm{~d} s \mid \phi^{\odot \odot}\right\rangle_{X^{\odot}},
$$

which is a natural extension of the pairing $\langle\bullet \mid \bullet\rangle_{X}$, in the sense that

$$
\left[\phi \mid \phi^{*}\right]=\left\langle\phi \mid \phi^{*}\right\rangle_{X} \quad\left(\phi \in X, \phi^{*} \in X^{*}\right),
$$

if $X$ is equipped with the prime norm. Instead of $(\Sigma)$ we consider the 'extended' system

$$
x^{\odot \odot}(t)=T^{\odot \odot}(t) \phi^{\odot \odot}+\int_{0}^{t} T^{\odot *}(t-s) B u(s) \mathrm{d} s .
$$

It is easy to check that, with the natural adaptions, all results of Sections 4 and 5 carry over to this case.

An important issue in control theory is stabilizability. The control system $(\Sigma)$ is called (exponentially) stabilizable if there exists a bounded linear operator $F: X \rightarrow U$ such that the semigroup $T_{F}(t)$ determined by

$$
T_{F}(t) \phi=T(t) \phi+\int_{0}^{t} T^{\odot *}(t-s) B F T_{F}(s) \phi \mathrm{d} s
$$

is of strictly negative type, i.e.

$$
\omega_{0}\left(A_{F}\right)<0
$$

(see Section 2 for a definition of type). This implies that there exists an $\varepsilon>0$ and $M>0$ such that

$$
\left\|T_{F}(t) \phi\right\| \leqslant M \mathrm{e}^{-\varepsilon t}\|\phi\|
$$

for every $t \geqslant 0$. In [14], Triggiani proves some results concerning the stabilizability of $(\Sigma)$ under the assumption that $B$ maps into $X$. Here we shall briefly indicate how his results carry over to the situation described in Section 4 . Let $P_{+}: X \rightarrow X$ be a bounded projection, and let

$$
X=X_{-} \oplus X_{+}
$$


be the corresponding decomposition of the state space $X$, i.e. $X_{+}=P_{+} X$, $X_{-}=P_{-} X$, where $P_{-}=I-P_{+}$. Assume that $X_{-}$and $X_{+}$are invariant under $T(t)$, and let $T_{-}(t)$ and $T_{+}(t)$ be the corresponding semigroups obtained by restriction. It is obvious that $P_{-}$and $P_{+}$commute with $T(t)$, and therefore (see [9]) we can extend these operators to $X^{\odot *}$; we denote these extensions by $P_{-}^{\odot *}$ and $P_{+}^{\odot *}$ respectively. Set

$$
B_{-}=P_{-}^{\odot *} B, \quad B_{+}=P_{+}^{\odot *} B .
$$

Then $B_{-}: U \rightarrow X_{-}^{\odot *}=P_{-}^{\odot *} X^{\odot *}$ and $B_{+}: U \rightarrow X_{+}^{\odot *}=P_{+}^{\odot *} X^{\odot *}$ are bounded linear operators. Now we can decompose the control system $(\Sigma)$ as follows:

$$
\begin{aligned}
& x_{-}(t)=T_{-}(t) \phi_{-}+\int_{0}^{t} T_{-}^{\odot *}(t-s) B_{-} x_{-}(s) \mathrm{d} s, \\
& x_{+}(t)=T_{+}(t) \phi_{+}+\int_{0}^{t} T_{+}^{\odot *}(t-s) B_{+} x_{+}(s) \mathrm{d} s,
\end{aligned}
$$

defined on $X_{-}$and $X_{+}$, respectively. The following result holds (see [12]).

THEOREM 7.1 If $T_{-}(t)$ is exponentially stable, i.e. $\omega_{0}\left(A_{-}\right)<0$, then the original system $(\Sigma)$ is exponentially stabilizable if and only if $\left(\Sigma_{+}\right)$is exponentially stabilizable.

The proof of this result is straightforward. One can think of the following application. Suppose that the (Browder) essential type $\omega_{\text {ess }}(A)$ of $T(t)$ (see [15]) is negative, say $\omega_{\text {ess }}(A)<-\delta$, then $\sigma(A) \cap\{\lambda \in \mathbb{C}: \operatorname{Re} \lambda \geqslant-\delta\}$ is finite, and contains only eigenvalues with finite algebraic multiplicity. Define $P_{+}$to be the spectral projection corresponding to the spectral set $\sigma(A) \cap\{\lambda \in \mathbb{C}: \operatorname{Re} \lambda \geqslant-\delta\}$. Then $\omega_{0}\left(A_{-}\right) \leqslant-\delta$, hence $T_{-}(t)$ is exponentially stable, and, moreover, $X_{+}$is finite-dimensional. Now, a famous result from finite-dimensional system theory (see e.g. [3]) says that $\left(\Sigma_{+}\right)$is exponentially stabilizable if it is controllable. We refer to [14] for a number of related results.

Finally, we suspect that the approach presented in this paper will appea] suitable for boundary control systems as well. This presumption is sustained by the example described in [2]. In this example, concerning the system describin! age-dependent population growth, the (non-local) boundary condition can br formulated abstractly by a bounded linear perturbation $C: X \rightarrow X^{\odot *}$.

\section{REFERENCES}

1. Butzer, P. L., \& Berens, H. 1967 Semigroups of Operators and Approximatior Springer, Berlin.

2. Clément, Ph., Diekmann, O., Gyllenberg, M., Heimmans, H. J. A. M., ، Thieme, H. R. 1986 Perturbation theory for dual semigroups. I. The sun-reflexiv case. CWI Report, AM-R86-05. Parts II-IV in preparation.

3. Curtain, R. F., \& Pritchard, A. J. 1977 Functional Analysis in Modern Applie Mathematics. Academic Press, London.

4. Desch, W., Lasiecka, I., \& Schappacher, W. 1985 Feedback boundary contr problems for linear semigroups. Israel J. Math. 51, 177-207. 
5. Diekmann, O. (to appear) Perturbed dual semigroups and delay equations. to appear in the proceedings of a meeting on infinite dimensional dynamical systems, Lisbon, 1986.

6. Dolecki, S., \& Russell, D. L. 1977 A general theory of observation and control. SIAM J. Control Optimiz. 15, 185-220.

7. Fattorini, H. O. 1966 Some remarks on complete controllability. SIAM J. Control Optimiz. 4, 686-694.

8. Fattorini, H. O. 1968 Boundary control systems. SIAM J. Control Optimiz. 6, 349-385.

9. Hille, E., \& Phillrips, R. S. 1957 Functional Analysis and Semigroups. Providence: AMS.

10. Manrtius, A. 1981 Necessary and sufficient conditions of approximate controllability for general linear retarded systems. SIAM J. Control Optimiz. 19, 561-632.

11. Manitius, A., \& Triggiani, R. 1978 Function space controllability of linear retarded systems: a derivation from abstract operator conditions. SIAM J. Control Optim. 16, $599-645$.

12. Pritchard, A. J., \& ZABCZYK, J. 1981 Stability and stabilizability of infinite dimensional systems. SIAM Rev. 23, 25-52.

13. Salomon, D. 1984 Control and Observation of Neutral Systems. Research Notes in Mathematics 91. London: Pitman.

14. Triggiani, R. 1975 On the stabilizability problem in Banach space. J. Math. Anal. Appl. 52, 383-403.

15. WebB, G. F. 1985 Theory of Nonlinear Age-dependent Population Dynamics. New York: Dekker. 